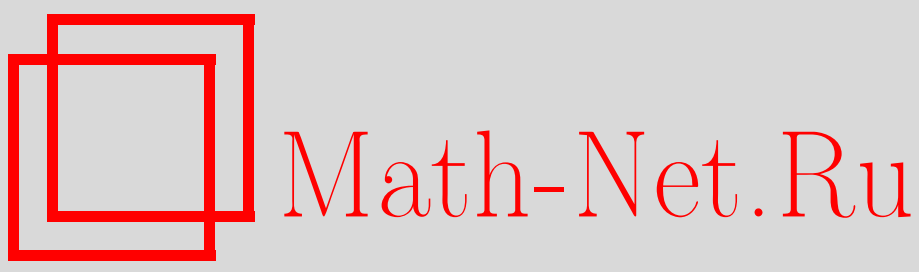

В. А. Акулов, Исследование гемодинамики нижних конечностей в условиях гипергравитации методами вычислительного эксперимента, Вестн. Сам. гос. техн. ун-та. Сер. Физ.-мат. науки, 2000, выпуск 9, 161-168

DOI: https://doi.org/10.14498/vsgtu43

Использование Общероссийского математического портала Math-Net.Ru подразумевает, что вы прочитали и согласны с пользовательским соглашением

http://www.mathnet.ru/rus/agreement

Параметры загрузки:

IP : 34.229 .108 .108

26 апреля 2023 г., 13:42:49

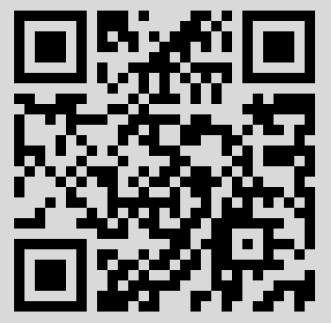


В. А. Акулов

\section{ИССЛЕДОВАНИЕ ГЕМОДИНАМИКИ НИЖНИХ КОНЕЧНОСТЕЙ В УСЛОВИЯХ ГИПЕРГРАВИТАЦИИ МЕТОДАМИ ВЫЧИСЛИТЕЛЬНОГО ЭКСПЕРИМЕНТА}

Выполнено математическое, аппаратное и техническое обеспечение в поддержку нового физиотерапевтического метода в травматологии и ортопедии - гравитационной терапии. Разработана соответствующчая концеепчия и структура информациионно-измерительного и вычислительного комплекса. Выполнены пилотные модельные и клинические испытания системыл.

Гравитационная терапия (ГТ) является новым физиотерапевтическим средством в травматологии и ортопедии $[1,2]$. Основу лечебного эффекта составляет принудительное нагнетание крови в нижние конечности, которое обеспечивается искусственной гравитацией, создаваемой в кранио-каудальном направлении («голова- ноги») специализированной центрифугой.

В результате дополнительного притока крови, а многие проблемы в травматологии и ортопедии связаны именно с недостаточным кровообращением пораженных участков, происходит «активное развитие коллатерального кровообращения, образование новых сосудов микроциркуляторного русла, что устраняет ишемию тканей, улучшает метаболизм» [2].

Эффективность ГТ, ближайшие и отдаленные перспективы ее развития в значительной степени зависят от уровня информационного обеспечения. В связи с этим выполнена серия исследований на междисциплинарном уровне (медицина- техника), и некоторые из полученных результатов приводятся в данной статье. В частности, впервые предложена концепция автоматизированной системы научных исследований в гравитащионной терапии (АСНИ ГТ) ${ }^{1}$.

\section{Постановка задачи}

Цель исследований - разработка концепции и структуры информационно-измерительного комплекса, оснащенного АСНИ, для лечебных центрифуг нового поколения

Задачи исследований следующие:

разработка блок- схемы информационно- измерительного комплекса, оснащенного автоматизированной системой научных исследований в гравитационной терапии (АСНИ ГТ);

разработка математической модели системы кровообращения нижних конечностей с учетом гравитации в кранио-каудальном направлении:

- построение расчетной схемы системы кровообращения конечностей;

- вывод дифференциальных уравнений относительного покоя элемента крови;

- построение силовой схемы, включающей в себя результирующую силу, приложенную к элементу крови, и ее составляющие;

- получение интегральных зависимостей инерционного напора и уровней перегрузки от локализации сечений и частоты вращения ротора (прямая и обратная задачи);

- проверка адекватности математической модели на фантомах ${ }^{2}$ системы кровообращения (методика проверки, экспериментальные исследования);

разработка программы с интерфейсом Windows, обеспечивающей технологию вычислительного эксперимента для целей практической и экспериментальной медицины, в их числе предпосылки для медико-технического обоснования проектов перспективных центрифуг и разработка подходов к выявлению скрытых закономерностей гемодинамики в условиях ГТ.

Предмет исследований - система кровообращения нижних конечностей человека в условиях искусственной гипергравитации ${ }^{3}$, создаваемой специализированной центрифугой короткого радиуса действия.

\footnotetext{
${ }^{1}$ Под АСНИ понимается человеко-машинная, программно-аппаратная система определенного целевого назначения, реализованная на базе средств вычислительной техники.

${ }^{2}$ Под фантомами понимаются физические модели системы кровообращения, смонтированные на центрифуге.

${ }^{3}$ Термин «гипергравитация» введен разработчиками ГТ для отличия естественной и искусственной гравитации.
} 


\section{Структура информационно - измерительного комплекса}

На рис. 1 представлена укрупненная блок-схема предлагаемого информационноизмерительного комплекса (ИИК ГТ).

Краткая характеристика каждого из информационных блоков приведена в табл. 1.

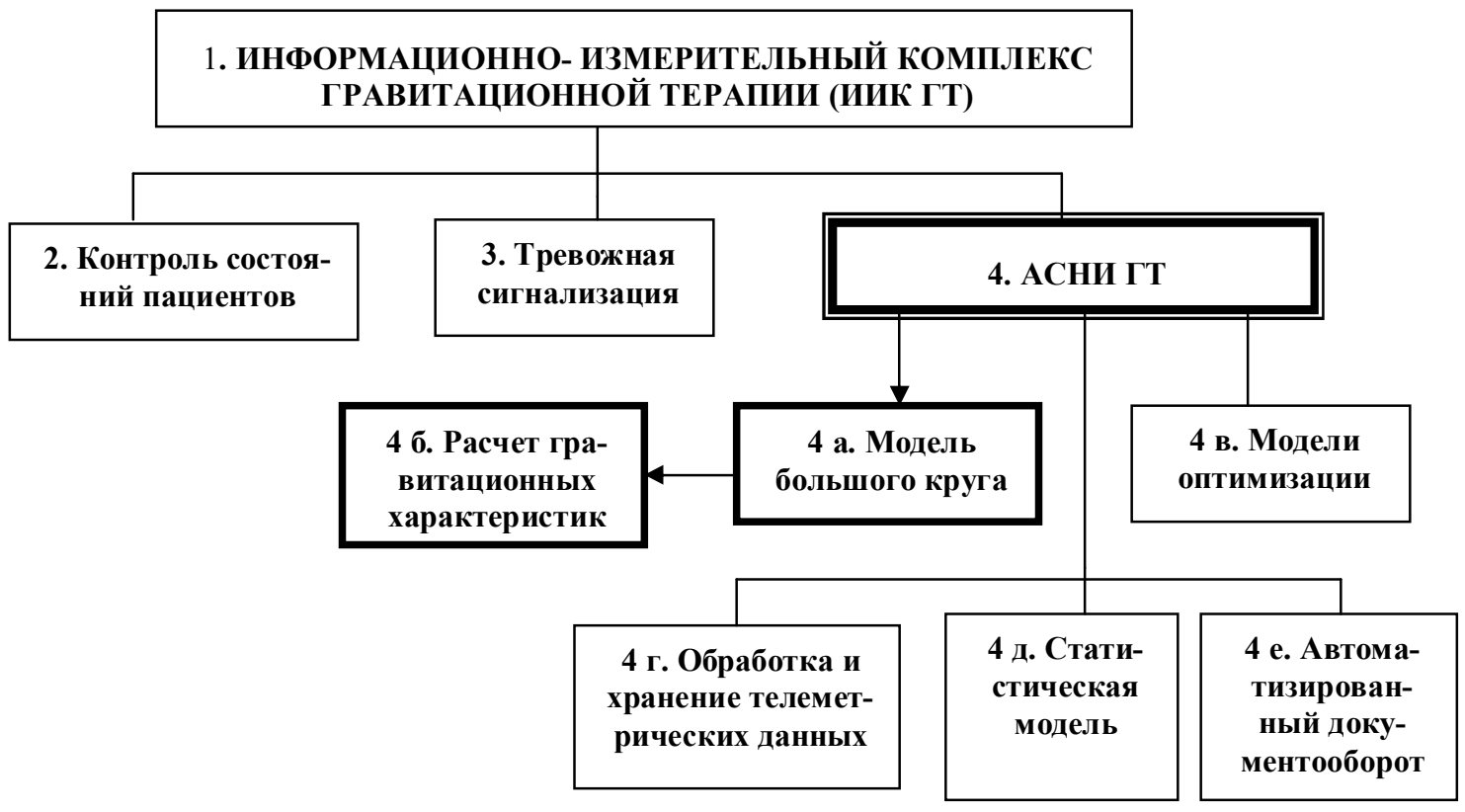

Р и с. 1. Блок- схема информационно-измерительного комплекса лечебной центрифуги

Таблица 1

Основные функции информационно- измерительного комплекса ГТ

\begin{tabular}{|c|c|c|}
\hline № II/II & Наименование & Функции \\
\hline 1 & \multicolumn{2}{|c|}{ ИНФОРМАЦИОННО- ИЗМЕРИТЕЛЬНЫЙ КОМПЛЕКС ГРАВИТАЦИОННОЙ ТЕРАПИИ } \\
\hline 2 & $\begin{array}{l}\text { Контроль состояний па- } \\
\text { циентов }\end{array}$ & $\begin{array}{l}\text { Измерение и регистрация номинального перечня медицинских } \\
\text { показателей }{ }^{4} \text {. Перечень параметров определяется врачом (АД, } \\
\text { ЧСС, ЭКГ, оксигенация тканей, работа мышц и т. д.) }\end{array}$ \\
\hline 3 & $\begin{array}{l}\text { Тревожная сигнализация } \\
\text { (Alarm) }\end{array}$ & $\begin{array}{l}\text { Выдача сигнала в случае критического состояния пациента, тре- } \\
\text { бующего прекращения терапии }\end{array}$ \\
\hline 4 & \multicolumn{2}{|r|}{ АСНИ В ГРАВИТАЦИОННОЙ ТЕРАПИИ } \\
\hline $4 \mathrm{a}$ & Модель большого круга & $\begin{array}{l}\text { Математическая модель системы кровообращения нижних ко- } \\
\text { нечностей в условиях ГТ }\end{array}$ \\
\hline 4 б & $\begin{array}{l}\text { Расчет гравитационных } \\
\text { характеристик }\end{array}$ & Решение прямой и обратной задачи (формулы (1- 4)) \\
\hline 4 в & Модели оптимизации & $\begin{array}{l}\text { Построение и применение индивидуализированной стратегии и } \\
\text { тактики лечения }\end{array}$ \\
\hline $4 \Gamma$ & $\begin{array}{l}\text { Обработка и хранение } \\
\text { телеметрических данных }\end{array}$ & $\begin{array}{l}\text { Измерение и регистрация расширенного перечня параметров } \\
\text { при экспериментах (пациенты, фантомы системы кровообраще- } \\
\text { ния) }\end{array}$ \\
\hline 4 д & Статистическая модель & $\begin{array}{l}\text { Описательная и индуктивная статистика: параметрический и } \\
\text { непараметрический анализ данных }\end{array}$ \\
\hline $4 \mathrm{e}$ & $\begin{array}{l}\text { Автоматизированный } \\
\text { документооборот }\end{array}$ & Электронный вариант эпикриза пациента \\
\hline
\end{tabular}

Наиболее важной особенностью ИИК ГТ является встроенная АСНИ (блок 4), которой посвящено дальнейшее изложение.

\footnotetext{
${ }^{4}$ Кроме номинального перечня предусмотрен расширенный перечень (см. позицию 4 г). 


\section{АСНИ ГТ. Прямая и обратная задачи}

Как показал опыт эксплуатации центрифуги, умеренные перегрузки в кранио-каудальном направлении обладают несомненным терапевтическим эффектом $[1,2]$. В то же время необходимо отметить, что механизмы лечения в значительной степени остаются неясными. Так, например, не дано объяснение отсутствия отеков конечностей в условиях повышенного артериального давления при ГТ. К числу важных проблем относится «оценка состояния дыхательной системы, динамики артериального и венозного давления, термометрия кожных покровов, уровень оксигенации тканей, тонометрия мышц (работа конечностей на тренажере в ходе ГТ)» и т. д. [2]. Все это требует выполнения значительного объема научно- исследовательских работ.

Кроме значительного числа измеряемых параметров и необходимости их оперативной обработки определенные затруднения создает специфика центрифуги (вращающийся ротор). Возникает проблема организации телеметрических каналов по схеме «борт- земля». Учитывая сказанное, автором предложена концепщия АСНИ в гравитационной терапии (см. рис. 1, табл. 1 поз. 4 - 4 e).

С точки зрения медицинской информатики принципиальное значение имеют блоки, связанные с моделированием и расчетом гравитационных характеристик (поз. 4 a, 4 б, выделенные на рис. 1 контурной линией). На них возложено решение двух задач, связанных с ГТ. Одна из них, получившая название прямой, заключается в вычислении прироста давления $(\Delta \mathrm{P})$, обусловленного действием центробежных сил, в зависимости от локализации (расстояния от оси вращения х) и частоты вращения ротора $(\omega)$, а также уровня перегрузок $[+G]$ от тех же аргументов:

$$
\begin{aligned}
& \Delta P=\Delta P(x, \omega) ; \\
& a[+G]=G(x, \omega) .
\end{aligned}
$$

Обратная задача состоит в определении частоты вращения $(\omega)$, обеспечивающей в заданном сечении $(x)$ заданный уровень прироста давления ( $[\Delta P])$ или заданный уровень перегрузки $([G+])$ :

$$
\begin{aligned}
& \omega=\omega(x,[\Delta P]), \\
& \omega=f\left(x,\left[G_{+}\right]\right) .
\end{aligned}
$$

\section{Математическая модель сосудистого русла конечностей}

\section{Расчетная схема}

Искусственная гравитация создается специализированной центрифугой, представляющей собой горизонтальный стол, который совершает вращательное движение вокруг вертикальной оси. Пациент размещается на столе в радиальном направлении с таким расчетом, чтобы голова располагалась на линии оси вращения, а конечности были обращены к периферии. Этим достигается снижение нагрузки на сердце и голову в сочетании с максимальным лечебным эффектом в нижних конечностях. Таким образом, с увеличением расстояния от оси растет инерционный напор крови. Эта специфика отражена в названии: «центрифуга короткого радиуса действия» (ЦКР) [2].

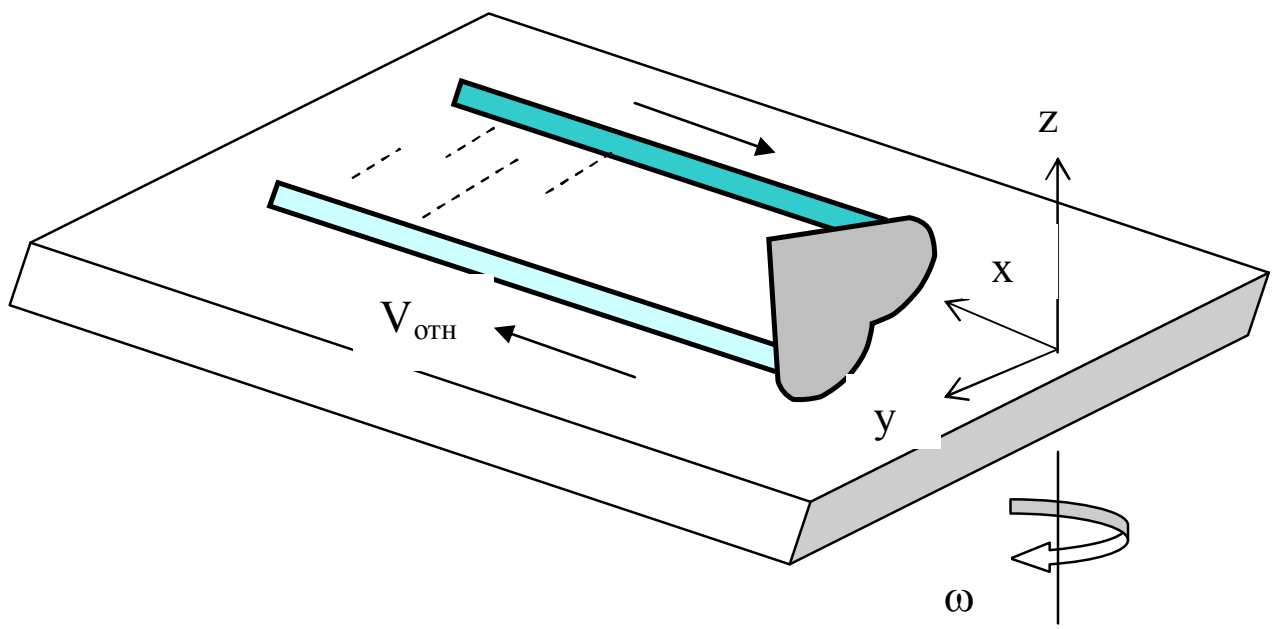

Р и с. 2. Расчетная схема системы кровообращения конечностей 
Что касается системы кровообращения конечностей, она обладает значительной анатомической сложностью и для решения поставленных задач нуждается в схематизации. Артериальная система представлена в виде эквивалентного сосудистого русла, расположенного на столе центрифуги радиально. По нему движется кровь в направлении от сердца к периферии со скоростью Vотн (рис. 2). Венозное русло также представлено одним сосудом, но с противоположным направлением течения.

Введем систему координат (x, y, z) и для определенности рассмотрим артериальное русло. Специфика ГТ такова, что с точки зрения теоретической механики каждый элемент совершает так называемое сложное движение [3, 4]. Во-первых, в результате насосной функции сердца элемент движется от центра к периферии со скоростью Уотн, т. е. участвует в относительном движении. Во-вторых, само русло совершает вращательное движение с угловой скоростью $\omega$. Такое движение называется переносным. В результате возникает так называемое поворотное (кориолисово) ускорение и соответственно кориолисова сила. К элементу также приложены поверхностные силы и сила тяжести. В итоге формируется 3- мерная расчетная схема.

\section{Дифференциальные уравнения гидростатики, результирующие ускорение и его компоненты}

Запишем уравнение гидростатики для общего случая (поверхностные и массовые силы), известное как уравнение Л. Эйлера [4]:

$$
X-\frac{1}{\rho} \frac{\partial p}{\partial x}=0, Y-\frac{1}{\rho} \frac{\partial p}{\partial y}=0, Z-\frac{1}{\rho} \frac{\partial p}{\partial z}=0 .
$$

Здесь $\mathrm{X}, \mathrm{Y}, \mathrm{Z}$ - проекции результирующей массовой силы на соответствующие оси в пересчете на единицу массы; $\rho$ - плотность; $р$ - давление; $\partial \bullet / \partial^{*}$ - оператор частного дифференцирования по соответствующему аргументу.

После несложных преобразований получим

$$
d p=\rho(X d x+Y d y+Z d z) .
$$

Подчеркнем, что уравнение (6) является дифференциальным уравнением равновесия жидкости в общем случае (сила тяжести и массовые силы инерции переносного движения). Таким образом, задача сводится к нахождению сил X, Y, Z, действующих в направлении соответствующих осей. Поскольку силы отнесены к единице массы, они имеют размерность ускорения $\left[\mathrm{M} / \mathrm{c}^{2}\right]$.

Как известно (теорема Г. Кориолиса [3]), в случае сложного движения точки результирующее ускорение (абс) равно геометрической сумме трех ускорений: относительного (отн), переносного (пер) и кориолисова (кор):

$$
\vec{a}_{\text {абс }}=\vec{a}_{\text {отн }}+\vec{a}_{\text {пер }}+\vec{a}_{\text {кор }} \text {. }
$$

Рассмотрим каждую из составляющих уравнения (7). Для ускорения в относительном движении имеем

$$
\vec{a}_{\text {oтн }}=d \vec{V}_{\text {отн }} / d t .
$$

Поскольку вариации относительной скорости малы по сравнению с окружными скоростями в первом приближении, полагаем $a_{\text {отн }}=0$.

В соответствии с расчетной схемой (горизонтальный стол, вращение вокруг вертикальной оси) переносное ускорение имеет две составляющие: радиальную (ось х) и тангенциальную (ось у):

$$
\vec{a}_{\text {nep }}=\vec{a}_{x}+\vec{a}_{y},
$$

где $a_{x}=x \cdot \varpi^{2}, x$ - текущий радиус точки (расстояние от оси вращения), ·ш- угловая скорость.

Учитывая значительную инерционность ротора (металлическая конструкция, пациент) и постоянство частоты вращения электропривода, пренебрегаем малыми изменениями угловой скорости. В итоге, тангенциальная составляющая ускорения $\mathrm{a}_{\mathrm{y}} \approx 0$. Тогда переносное ускорение направлено по радиусу к началу координат, а центробежная сила, приложенная к элементу, имеет противоположное направление и рассчитывается по формуле

$$
a_{\text {nep }}=x \cdot \omega^{2} \text {. }
$$

Что касается кориолисова ускорения, оно находится как векторное произведение $a_{\text {кор }}=2\left[\varpi V_{\text {отн }}\right]$, следовательно, $/ a_{\text {кор }} /=2 \omega V_{\text {отн }} \sin \left(\omega^{\wedge} V_{\text {оти }}\right)$. 
Так как $\sin \left(\omega^{\wedge} V_{\text {отн }}\right)=1$ (векторы ортогональны), заключаем следующее:

$$
\left|a_{\text {кор }}\right|=2 \omega V_{\text {отн. }}
$$

Необходимо учесть, что в вертикальном направлении действует сила тяжести, которая в пересчете на единицу массы определится как

$$
Z=-g \text {. }
$$

Обобщая полученные результаты (8)- (11), имеем:

$$
X=x \cdot \omega^{2}, \quad Y=2 \omega V_{\text {отн }} ; \quad Z=-g .
$$

Представим выражение (12) графически в виде силовой схемы (рис. 3), где F- результирующая сила, приложенная к элементу крови в пересчете на единицу массы.

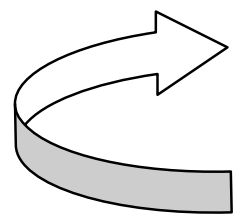

$\omega$

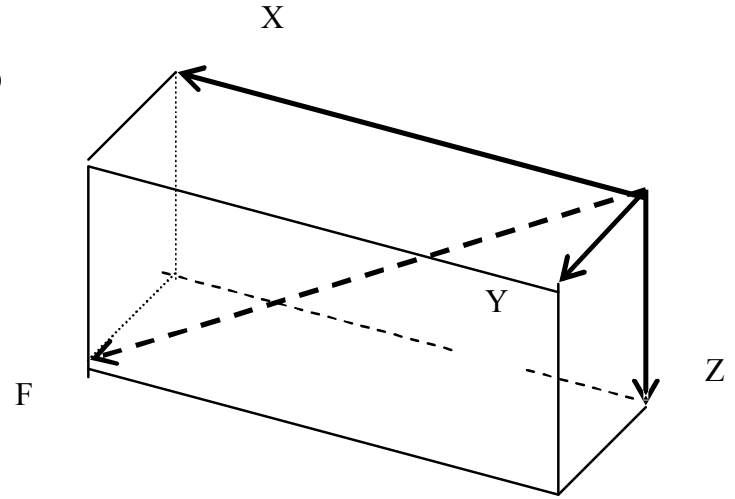

Р и с.3. Силы, действующие на произвольный

элемент крови в артериальном русле

Как видно, кориолисова сила (Y) осуществляет прогиб артериального русла в направлении, противоположном направлению вращения (показано стрелкой). Для венозного русла - картина обратная (относительные скорости противонаправлены).

Таким образом, в условиях ГТ возникает весьма специфическое явление, заключающееся в формировании сил, направленных тангенциально и «разводящих» артериальное и венозное русло в противоположные стороны. До определенных пределов это явление не представляет опасности ${ }^{5}$ Что касается опасных пределов, их нахождение является самостоятельной, технически сложной задачей.

Особо отметим, что полученные результаты следует рассматривать как оценочные и как приоритетные попытки выявления наиболее общих закономерностей гемодинамики методами вычислительного эксперимента. Что касается уточненных данных, необходимы прямые эксперименты с измерением пульсаций скорости в сосудистых руслах при работающей ЦКР. Такая задача не имеет ближайших аналогов, технически весьма сложна, но она запланирована на перспективу.

\section{Расчет инерционного напора (прямая задача)}

Одной из важнейших задач ГТ, возложенных на АСНИ, является расчет прироста давления, обусловленного вращением (см. формулу (1)). Для получения расчетной формулы запишем (6) для одномерного случая с учетом (9)

$$
d P=\rho \cdot x \cdot \omega^{2} d x
$$

Проинтегрируем (13) в пределах от $\mathrm{R}_{1}$ до $\mathrm{R}_{2}$, где $\mathrm{R}_{1}$ - некоторое начальное сечение русла ${ }^{6}$, $\mathrm{R}_{2}$ - расстояние по оси х до интересующего сечения, м:

$$
\Delta P=\rho \cdot \omega^{2}\left(R_{2}^{2}-R_{1}^{2}\right) / 2 .
$$

Преобразуем (14) к размерностям, принятым в медицине (АД- в мм. рт. столба (Н), частота вращения ротора в об / мин (n)):

$$
\Delta H=4086,6667 \cdot \rho \cdot \pi^{2} \cdot n^{2} \cdot\left(R_{2}^{2}-R_{l}^{2}\right) .
$$

Из (15) следует, что инерционный напор крови, обусловленный вращением, существенно (во второй степени) зависит как от текущего радиуса $\mathrm{R}_{2}$, так и от частоты вращения ротора

\footnotetext{
${ }^{5}$ Анатомически сосуды тесно переплетены и надежно закреплены.

${ }^{6}$ Если начальное сечение совпадает с осью вращения $\mathrm{R}_{1}=0$.
} 
ЦКР. В табл. 2 в качестве примера приведены расчетные значения инерционного напора $(\Delta \mathrm{H})$ для двух характерных сечений $\mathrm{R}_{2}$.

Таблица 2

Примеры расчетных значений $\Delta \mathrm{H}, \mathbf{a}[+\mathrm{G}]$, режим $\mathbf{n}=33$ об / мин

\begin{tabular}{|l|l|l|l|}
\hline $\mathbf{R}_{\mathbf{2}}, \mathbf{M}$ & $\mathbf{\Delta} \mathbf{H}, \mathbf{м м .} \mathbf{p т .} \mathbf{c \mathbf { T }}$ & $\mathbf{a}[+\mathbf{G}]$ & Примечание \\
\hline 1,0 & 42,1 & 1,22 & Бедро \\
\hline 1,5 & 100,5 & 1,82 & Голень \\
\hline
\end{tabular}

Как видно, инерционный напор соизмерим с уровнем АД, однако в ходе ГТ пациенты его не ощущают. Объясняется это тем, что суммарное давление остается в физиологических пределах, характерных для мышечной работы.

\section{Расчет перегрузок в произвольном сечении конечностей}

Воспользуемся формулой (9). Учитывая, что $\omega=\pi n / 30$, а $x=R_{2}$, переходя к безразмерному виду делением на ускорение свободного падения $g$, получим

$$
a[+G]=\pi^{2} n^{2} R_{2} / 900 \mathrm{~g} .
$$

В качестве примера в табл. 2 приведены уровни перегрузки для двух характерных сечений. В частности, в районе голени перегрузки становятся значительными, достигая почти двукратного уровня.

\section{Обратная гравитационная задача}

В общем виде обратная задача ГТ представлена формулами (3), (4). Чтобы получить расчетные формулы для АСНИ, достаточно разрешить (15) и (16) относительно частоты вращения (n). В целях сокращения окончательные соотношения здесь не приводятся.

\section{Проверка адекватности математической модели}

Проверка заключалась в сравнении расчетных данных по параметру $\Delta H$ с экспериментальными, полученными при испытании фантомов системы кровообращения (упрощенных физических моделей) на центрифуге. Для осуществления проверки была разработана методика, отвечающая следующим требованиям: простота реализации, наглядность, высокая достоверность результатов, низкая стоимость. На рис. 4 показана конструктивная схема фантома. Как видно, она отличается исключительной простотой и включает в себя бачок 1 ; трубку 2 , имитирующую сосудистое русло, и пьезометры $3,4,5^{7}$.

Бачок частично заполнен водой, сообщается с атмосферой и устанавливается на линии оси вращения. Его назначение- подпитка элементов 2- 5 водой и обеспечение постоянства давления в начальном сечении имитатора 2.

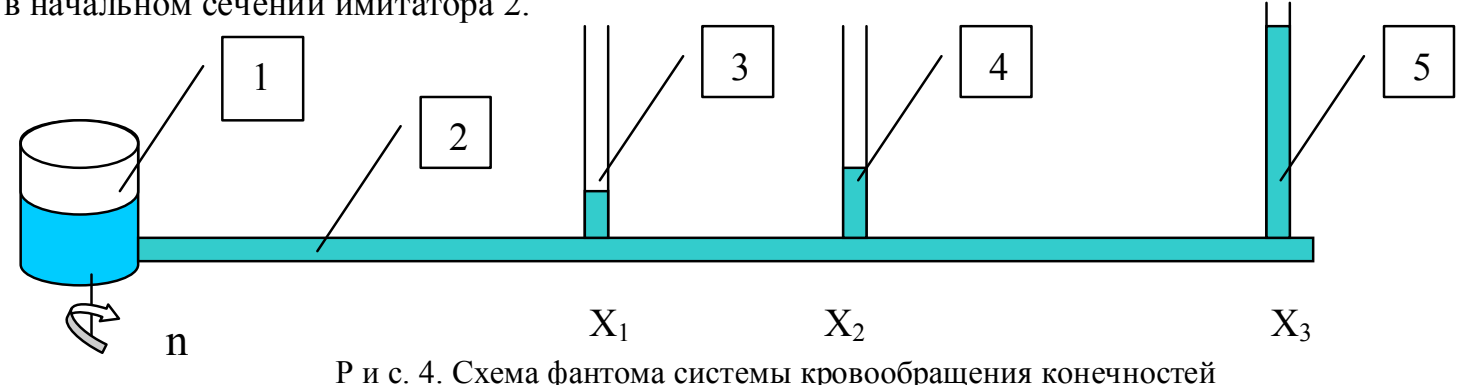

Пьезометры и имитатор были изготовлены из пластмассовой трубки, применяемой в капельницах. Поскольку напор жидкости существенно (в квадрате) зависит как от локализации

\footnotetext{
${ }^{7}$ Пьезометр представляет собой прозрачную вертикальную трубку, один конец которой подключается к точке измерения напора, а другой- сообщается с атмосферой. Для измерения высоты столба жидкости служит мерная линейка [4]. 
сечения, так и частоты вращения ротора, были приняты меры по точному измерению расстояний до точек $\left(\mathrm{X}_{1}, \mathrm{X}_{2}, \mathrm{X}_{3}\right)$ и выполнена тарировка тахометра ${ }^{8}$.

Опуская технические подробности, отметим, что максимальное расхождение расчетных и экспериментальных данных не превышало 8 см водяного столба при номинальной высоте столба в пьезометре порядка $150 \mathrm{~cm}$. Таким образом, относительная погрешность составила около 2 $\%$, что свидетельствует об адекватности математической модели.

Кроме проверки адекватности на фантомы возлагается целый ряд задач, связанных с перспективой. В их числе медико-техническое обоснование конструкции ЦКР нового поколения (компоновка, силовой привод, технологическая безопасность) и расширение функций ИИК ГТ. Другой задачей является выявление скрытых механизмов ГТ. В этой связи расчетные данные и данные, полученные при экспериментах с фантомами, являются базовыми для оценки регуляторных функций организма. В частности, с помощью препаратов можно изменять насосную функцию сердца, влиять на компенсаторные реакции и т. п., а затем оценивать их влияние путем сравнения результатов на живом организме и фантомах.

\section{Программный интерфейс АСНИ ГТ}

Одной из важнейших функций АСНИ является расчет гравитационных характеристик (см. поз. 4 б, рис.1). На рис. 5 показан типовой экран интерфейса, который состоит из 4 областей. В правом верхнем углу осуществляется ввод исходных данных: рост, положение головы и т. д. Три остальные области отведены под вывод расчетных данных (формулы (15), (16), прямая задача). В правом нижнем углу данные выводятся в табличном виде с шагом по длине, равным 10 см, что вполне достаточно для локализации сечений конечностей.

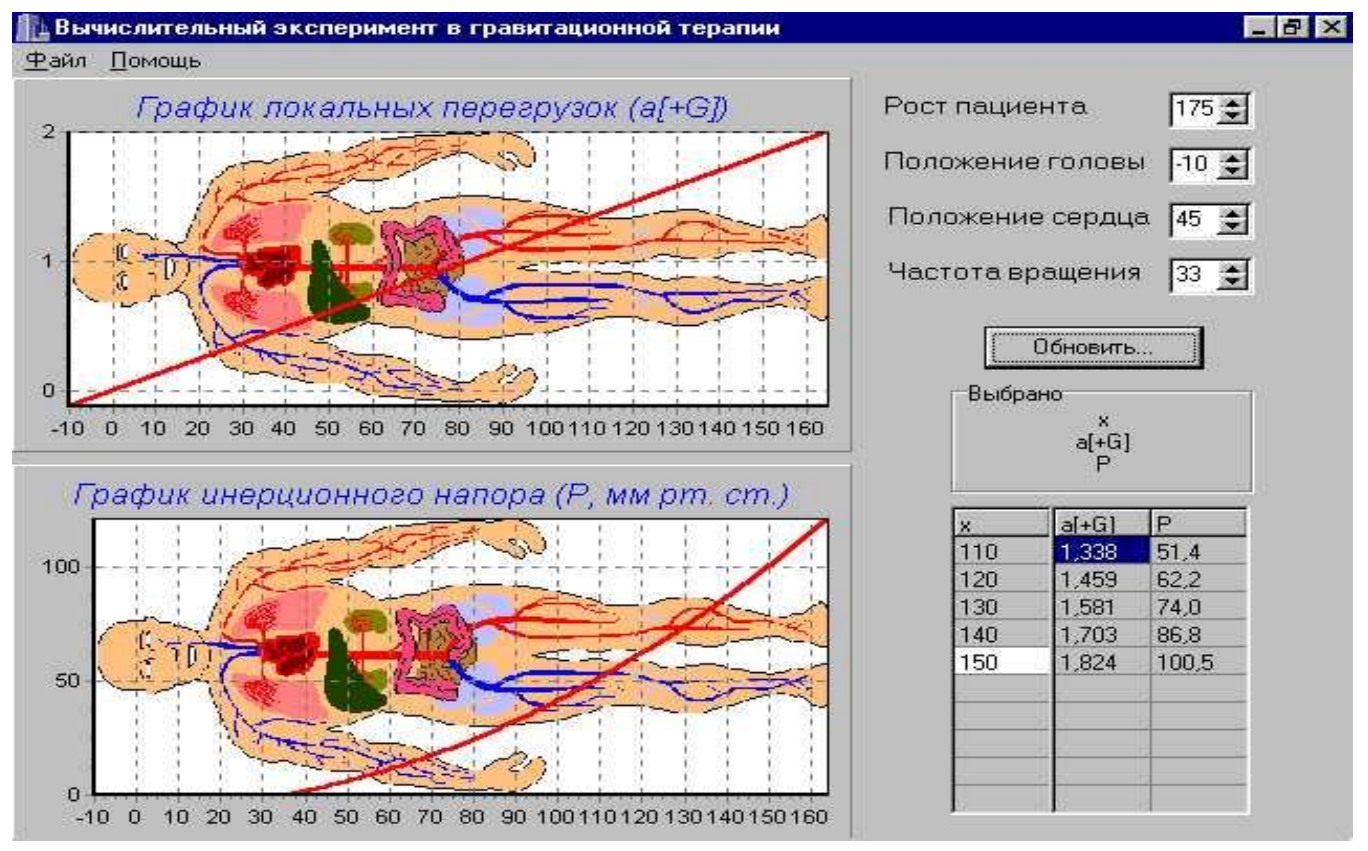

Р и с. 5. Пример типового экрана АСНИ ГТ (гравитационные характеристики)

Наиболее удобным для медицинского пользователя является графическое представление, где в качестве фона применена анатомическая схема человека. Верхний рисунок иллюстрирует распределение перегрузок, а нижний- инерционных напоров. Важно отметить, что графики автоматически масштабируются в соответствии с индивидуальными антропометрическими данными пациента. В случае необходимости, например для приобщения к истории болезни (эпикризу), предусмотрена распечатка данных.

Что касается обратной задачи (формулы (3),(4)), она временно решается методом подбора параметра. По окончании отладки программы выбор интересующего сечения с заданными гравитационными характеристиками будет осуществляться «щелчком» мыши.

\footnotetext{
${ }^{8}$ Как выяснилось, на этапах, предшествовавших математическому моделированию, в качестве тахометра применялся вольтметр с грубой градуировкой.
} 
По заключению специалистов по ГТ, программное обеспечение заслуживает высокой оценки.

Из вышеизложенного можно сделать следующие выводы.

1. Разработана структура иформационно-измерительного комплекса, ориентированного на проблемы гравитационной терапии. Обоснована необходимость и разработана блок-схема автоматизированной системы научных исследований в гравитационной терапии (АСНИ ГТ).

2. Сформирована 3- мерная расчетная схема системы кровообращения конечностей в виде эквивалентных русел, совершающих сложное движение (относительное и переносное).

3. На основе уравнений Эйлера выведены дифференциальные уравнения относительного покоя элемента крови в условиях гипергравитации. Получены инженерные формулы расчета гравитационных характеристик (15), (16) (прямая и обратная задачи).

4. Построена 3- мерная силовая схема нагружения элемента крови и на ее основании установлен специфический для ГТ эффект «разнесения» артериального и венозного русел в тангенциальном направлении.

5. Разработан программный интерфейс, обеспечивающий режим вычислительного эксперимента в ГТ (прямая и обратные задачи). С целью наглядности в качестве фона графиков распределения перегрузок и инерционного напора по длине применены анатомические схемы человека. Масштаб схем автоматически изменяется в соответствии с индивидуальными данными пациента.

6. Разработана и начата реализация программы экспериментальных исследований на фантомах (упрощенных физических моделях) системы кровообращения. В частности, выполнена проверка адекватности модели сосудистых русел. Расхождение расчетных и экспериментальных данных не превышало $2 \%$.

7. В результате экспериментов на моделях и фантомах собраны основные исходные данные для медико-технического обоснования проектов перспективных ЦКР и выявления скрытых закономерностей гемодинамики в условиях ГТ.

\section{БИБЛИОГРАФИЧЕСКИЙ СПИСОК}

1. Котельников Г. П. Повышенная гравитационная нагрузка в системе реабилитационных мероприятий у травматолого-ортопедических больных // VI съезд травматологов-ортопедов России: Тез. докл. Н-Новгород, 1997. С. 820.

2. Яшков $A$. B. Клинико-экспериментальное обоснование применения гравитационной терапии у больных с нарушением репаративного остеогенеза нижних конечностей: Дисс. д-ра мед. наук. Самара, 1998.

3. Тарг С. М. Краткий курс теоретической механики. М: Высш. школа, 1986. 416 с.

4. Башта Т. М. и др. Гидравлика, гидравлические машины и гидравлические приводы. М.: Машиностроение, 1970. $504 \mathrm{c}$ 